\title{
"
}

\section{La Alianza del Pacífico+4 Y LA ESPECIALIZACIÓN REGIONAL DE COLOMBIA: \\ UNA APROXIMACIÓN DESDE LAS VENTAJAS COMPARATIVAS*}

\author{
Jenny Paola Danna-Buitrago
}

doi.10.11144/Javeriana.cao.30-55.aperc. Este artículo es producto de la investigación "Observatorio Colombiano de Tratados Comerciales (OCTC) - Fase III", contó con la financiación de la Fundación Universitaria los Libertadores. La autora agradece al Doctor Rémi Stellian por sus aportes, sin atribuirle ninguna responsabilidad respecto al contenido del artículo. El artículo se recibió el 01/11/2017 y se aprobó el 20/12/2017. Sugerencia de citación: Danna-Buitrago., J. P. (2017). La Alianza del Pacífico+4 y la especialización regional de Colombia: una aproximación desde las ventajas comparativas. Cuadernos de Administración, 30(55), 163-192. http://dx.doi.org/10.11144/Javeriana.cao.30-55.aperc.

* * Doctora en Ciencias Económicas de la Université de Grenoble, Grenoble, Francia, 2012. Directora de investigación en globalización y Desarrollo Sostenible en la Fundación Universitaria Los Libertadores, Bogotá, Colombia; Directora del Observatorio Colombiano de Tratados Comerciales (OCTC) y del grupo de Investigación Reflexión Económica y Contable de la misma Institución.

Correo electrónico: jenny.danna@libertadores.edu.co / jenny.sdanna@gmail.com 


\section{La Alianza del Pacífico+4 y la especialización regional de Colombia: una aproximación desde las ventajas comparativas}

\section{RESUMEN}

Este artículo explora cómo la extensión de la Alianza del Pacífico (AP) a Australia, Canadá, Nueva Zelandia y Singapur afectaría la especialización regional de Colombia. Se calcula el indicador de Ventajas Comparativas Reveladas (VCR) en términos de Contribución al Saldo Comercial (CSC) para Colombia en dos zonas de intercambios: la AP y la Alianza del $\mathrm{Pac}$ fíco+4 $(\mathrm{AP}+4)$. Pasando de la primera zona a la segunda, más productos pierden sus ventajas comparativas respecto a los productos que adquieren ventajas. Así, la $\mathrm{AP}+4$ implicaría más amenazas que oportunidades para la especialización regional de Colombia, y afectaría principalmente los productos alimenticios, los artículos manufacturados y la maquinaria y equipo de transporte.

Palabras claves: Alianza del Pacífico, Alianza del Pacífico+4, Colombia, especialización regional, ventajas comparativas reveladas, estacionariedad tendencial en el tiempo. Clasificación JEL: F13, F15, F63

\section{The Pacific Alliance+4 and \\ Regional Specialization in Colombia: An approach from the comparative advantages}

\section{AbSTRACT}

This article explores how the extension of the Pacific Alliance (PA) to Australia, Canada, New Zealand and Singapore would affect Colombia's regional specialization. The indicator of Revealed Comparative Advantages (RCA) is calculated in terms of Contribution to the Commercial Balance (CCB) for Colombia in two exchange zones: the PA and the Pacific Alliance +4 (PA $+4)$. Moving from the first zone to the second one, more products lose their comparative advantages with respect to the products that acquire advantages. Thus, PA +4 would involve more threats than opportunities for regional specialization in Colombia, and would mainly affect food products, manufactured goods and transport machinery and equipment. Previously, the use of the RCA-CCB indicator is justified because of its better stationary trend in time, compared with other representative RCA indicators.

Keywords: Pacific Alliance, Pacific Alliance+4, Colombia, Regional Specialization, Revealed Comparative Advantages

JEL Codes: $\mathrm{F}_{13}, \mathrm{~F}_{15}, \mathrm{~F}_{3}$

\section{A Aliança do Pacífico+4 e a especialização regional da Colômbia: uma aproximação a partir das vantagens comparativas}

\section{ResUMO}

Este artigo explora como a extensão da Aliança do Pacífico (AP) à Austrália, ao Canadá, à Nova Zelândia e a Singapura afetaria a especialização regional da Colômbia. Calcula-se o indicador de Vantagens Comparativas Reveladas (VCR) em termos de Contribuição para o Saldo Comercial (CSC) para a Colômbia em duas zonas de intercâmbios: a AP e a Aliança do $P a c i ́ f i c o+4(A P+4)$. Passando da primeira zona à segunda, mais produtos perdem suas vantagens comparativas a respeito dos produtos que adquirem vantagens. Assim, a $\mathrm{AP}+4$ implicaria mais ameaças que oportunidades para a especialização regional da Colômbia e afetaria principalmente os produtos alimentares, artigos manufaturados e maquinaria, bem como equipamento de transporte.

Palavras-chave: Aliança do Pacífico, Aliança do Pacífico+4, Colômbia, especialização regional, vantagens comparativas reveladas, estacionariedade tendencial no tempo.

Classificação JEL: F13, F15, F63 


\section{Introducción}

La Alianza del Pacífico es un acuerdo de integración regional que suscribieron Chile, Colombia, México y Perú, mediante la llamada Declaración de Lima del 28 de abril de 2011. Estos cuatro países conciben la Alianza del Pacífico como "un mecanismo de articulación política, económica, de cooperación e integración que busca encontrar un espacio para impulsar un mayor crecimiento y mayor competitividad" a través de "un avance progresivo de la libre circulación de bienes, servicios, capitales y personas". ${ }^{1}$

Este énfasis sobre la eliminación de los obstáculos a los flujos económicos regionales se encuentra en el Acuerdo Marco de la Alianza del Pacífico, cuyo preámbulo resalta que los miembros están

convencidos que la integración económica regional constituye uno de los instrumentos esenciales para que los Estados de América Latina avancen en su desarrollo económico y social sostenible, promoviendo una mejor calidad de vida para sus pueblos y contribuyendo a resolver los problema que aún afectan a la región, como son la pobreza, la exclusión y la desigualdad social persistentes. (p. 2) ${ }^{2}$

En particular, los miembros están avanzando hacia la conformación de una zona de libre comercio, suprimiendo poco a poco los obstáculos tarifarios y no-tarifarios a los intercambios. Al respecto, el Acuerdo Marco arriba mencionado y el documento denominado "Protocolo Adicional" contienen las reglas que Chile, Colombia, México y Perú deben aplicar con el objetivo de estimular la liberalización del comercio en el marco de la Alianza del Pacífico.

A la vez que los cuatro países promueven el bloque comercial dentro de la Alianza del Pacífico, existe la iniciativa para extender este bloque hacia otras partes del mundo, especialmente la región Asia-Pacífico, ya que la Alianza del Pacífico considera esta región como fundamental en la economía global. Bajo esta óptica, la Declaración de Cali del 30 de junio de 2017, además de reiterar el compromiso de los miembros con el libre comercio, establece que se abren negociaciones con Australia, Canadá, Nueva Zelandia

1 Sitio Internet de la Alianza del Pacífico: alianzapacifico.net/que-es-la-alianza/\#la-alianza-del-pacifico-ysus-objetivos.

2 El acuerdo marco se puede consultar en el sitio internet de la Alianza del Pacifico: https://alianzapacifico. net/download/acuerdo-marco-de-la-alianza-del-pacifico/\# 
y Singapur para implementar acuerdos comerciales e incluso tratados de libre comercio. Una primera ronda de negociaciones con representantes de los ocho países tuvo lugar en octubre de 2017, con el propósito de avanzar hacia la Alianza del Pacífico+4.

El presente trabajo analiza cómo la transición de la Alianza del Pacífico a la Alianza del Pacífico+4 influiría sobre la especialización regional de Colombia, es decir, la concentración de la producción nacional en algunas categorías específicas de mercancías, con el fin de exportarlas hacia una zona específica de intercambios y así contribuir a la generación de ingresos vía la conformación de excedentes comerciales. El objetivo de este tipo de estudios es proporcionar resultados útiles para las entidades públicas encargadas de promover el desarrollo económico de Colombia en general, y para los participantes a las negociaciones arriba mencionadas en particular. Igualmente, estos resultados tienen el potencial para contribuir a la mejor comprensión de las implicaciones de la Alianza del Pacífico+4 por parte de las empresas colombianas involucradas en los intercambios regionales.

En la literatura no existen trabajos que estudien el tema del presente artículo; los trabajos existentes se centran en realizar análisis comparativos entre la Alianza del Pacífico y el Mercado Común del Sur -Mercosur- (Lámbarry, 2016; Martínez-C., 2016; Mora, 2016; González, 2017) o en hacer comparaciones entre la Alianza del Pacífico y otros acuerdos de integración regional en América Latina, como la Asociación Latinoamericana de Integración -ALADI- y la Unión de Naciones Suramericanas -UNASUR- (Maldonado y López, 2016; Quiliconi y Salgado, 2017). Igualmente, se encuentran investigaciones que estudian la Alianza del Pacífico en relación con la innovación (Arredondo et ál., 2016; García de Lema et ál., 2016), la inversión extranjera (Cocha y Gómez, 2016), los mercados de capitales (Arbeláez y Rosso, 2016), el impacto del precio del petróleo sobre la producción (Alonso y Martínez, 2017) y el capital humano (Castillo, 2016). Otros trabajos analizan la evolución del comercio en el marco de la Alianza del Pacífico, incluso algunos de ellos se interesan en evaluar cómo Colombia se vería afectada por el acuerdo (Gutiérrez et ál., 2013; Montoya et ál., 2016; Reyes y Medina, 2016; Ovando et ál., 2017; Ramírez et ál., 2017).

Sin embargo, la literatura no incluye trabajos que estudien el impacto de la conformación de la Alianza del Pacífico+4 sobre la especialización regional de Colombia. Si bien Tremolada (2014) analiza las posibilidades de inserción de Colombia en la región Asía-Pacífico, su estudio no se focaliza en la creación de la Alianza del Pacífico+4 y los posibles efectos de la extensión de la Alianza del Pacífico sobre la especialización regional de Colombia. 
Para resumir, la literatura aún no ha estudiado la Alianza del Pacífico+4 en general y los desafíos que su conformación generaría para la especialización regional de Colombia en particular. Por esta razón, el presente trabajo pretende llenar este vacío en la literatura.

Más allá de su interés para los practitioners, este artículo pretende aportar lineamientos metodológicos útiles para analizar la integración de un país a los flujos comerciales internacionales. Por una parte, el efecto de la Alianza del Pacífico+4 sobre la especialización regional de Colombia se estudia a partir del indicador denominado Ventajas Comparativas Reveladas -VCR-. Por otra parte -y aquí está el aporte metodológico de esta investigación- se resaltan evidencias a favor de un indicador VCR poco utilizado en la literatura, a pesar de caracterizarse por un mejor nivel de precisión. Se trata del indicador VCR en términos de Contribución al Saldo Comercial -CSC-. En este sentido, se señala que en ninguno de los trabajos existentes en la literatura sobre la Alianza del Pacífico y la Alianza del Pacífico+4 se realiza un análisis de las ventajas comparativas de Colombia mediante el indicador VCR-CSC, por lo que también se llena un vacío en la literatura.

El artículo se divide en cuatro secciones. La primera sección presenta de manera general en qué consiste un indicador VCR, por qué resulta útil para la presente investigación y cómo se calcula el indicador VCR-CSC. De acuerdo con esto, la segunda sección justifica por qué el indicador VCR-CSC se considera como la mejor opción para este estudio, a partir de una comparación con otros indicadores VCR disponibles en la literatura. Esta sección será la oportunidad de introducir los parámetros de cálculo de cada indicador VCR, incluso el indicador VCR-CSC. Finalmente, en la tercera sección se analizan los cálculos en cuestión, con la finalidad de hallar resultados respecto a la influencia de la Alianza del Pacífico+4 sobre la especialización regional de Colombia.

\section{Ventajas comparativas e indicador de Ventajas Comparativas Reveladas (VCR) en términos de Contribución al Saldo Comercial (CSC)}

Un indicador Ventajas Comparativas Reveladas -VCR- tiene como finalidad poner en evidencia ("revelar") en qué medida un país dispone de ventajas comparativas respecto a los demás socios comerciales dentro de una zona de intercambios determinada (Balassa, 1965; Vollrath, 1991). El concepto de (des)ventajas comparativas hace referencia a la (in)capacidad de producir un bien o servicio a menores costos relativos y con mayor diferenciación (Palley, 2008). Un país puede especializarse en los bienes y servicios 
caracterizados por poseer ventajas comparativas, adquiriendo así el potencial para favorecer la producción a través de los intercambios en la zona considerada. Lo contrario ocurriría en el caso de poseer desventajas comparativas.

En este contexto, resulta útil calcular un indicador VCR para Colombia en el marco de dos zonas de intercambios internacionales: i) la zona "inicial" conformada por los miembros de la Alianza del Pacífico; y ii) la zona "final" que incluye Australia, Canadá, Nueva Zelandia y Singapur además de los miembros de la Alianza del Pacífico. En efecto, si por ejemplo el indicador VCR de un bien/servicio revela ventajas comparativas en lugar de desventajas comparativas pasando de la zona inicial a la zona final, entonces se puede inferir que la liberalización del comercio hacia Australia, Canadá, Nueva Zelandia y Singapur abre nuevas oportunidades de especialización regional para Colombia. Recíprocamente, si el indicador VCR revela desventajas comparativas en lugar de ventajas comparativas desde la zona Alianza del Pacífico hacia la zona Alianza del Pacífico+4, entonces la incorporación de los cuatro países al bloque comercial inicialmente conformado podría cuestionar la especialización regional de Colombia. En ambos ejemplos, se trata de realizar un análisis comparativo entre los indicadores VCR de cada zona, con la finalidad de inferir cómo la extensión de la Alianza del Pacífico incitaría a considerar nuevamente la estructura de producción y de intercambios de Colombia.

Para ello, es necesario precisar cómo se calculan indicadores VCR para la presente investigación. Al respecto, se resaltan cuatro propiedades que caracterizan un indicador VCR (Yeats, 1985; Lafay, 1992):

1. Un indicador VCR se calcula a partir de datos sobre los intercambios, es decir, el indicador "revela" las ventajas/desventajas con base en la estructura de las exportaciones y las importaciones.

2. Un indicador VCR toma la forma de un único número real que sintetiza el nivel de ventajas/desventajas que muestra un país en cuanto a un bien/servicio durante un cierto periodo, al interior de una zona predeterminada de intercambios.

3. Existe un valor "neutro" (usualmente 0 o 1) que implica la inexistencia de ventajas/desventajas. Si un indicador VCR se ubica por debajo del valor neutro, esto denota la existencia de desventajas comparativas. Si un indicador VCR se ubica por encima del valor neutro, esto señala la existencia de ventajas comparativas. 
4. Por lo tanto, un indicador VCR mayor significa que las ventajas comparativas son mayores, o que las desventajas comparativas son menores, o que las desventajas son reemplazadas por ventajas; y vice-versa con un indicador VCR menor.

Acá se utiliza el indicador VCR en términos de Contribución al Saldo Comercial (CSC) (De Saint Vaulry, 2008), en donde: $i$ denota un país de la zona $J$ de intercambios, $k$ un producto de una nomenclatura $K$ y $t$ un periodo dentro de un conjunto $T$ de varios periodos. $X_{k i t}$ representa las exportaciones del producto $k$ realizadas por el país $i$ en el periodo $t ; y$ $M_{k i t}$ simboliza las importaciones de $k$ por $i$ en $t$. El cálculo del indicador VCR-CSC se puede presentar en cuatro etapas. Como primera etapa, se calcula el peso de $k$ en el comercio total de la zona en $t$, denotado $w_{k t}$ :

$$
W_{k t}=\frac{\sum_{i \in j}\left(X_{k i t}+M_{k i t}\right)}{\sum_{i \in J} \sum_{p \in K}\left(X_{p i t}+M_{p i t}\right)}
$$

$w_{k t}$ es el ratio de: i) el total, en $t$, de los flujos comerciales que involucran $k$ entre los diferentes países pertenecientes a $\mathrm{J}$; y ii) el total, en $t$, de los flujos comerciales que involucran todos los productos pertenecientes a $K$ entre los diferentes países pertenecientes a J. Por ejemplo, si $w_{k t}=0,1$ entonces $k$ representa el $10 \%$ del comercio de $J$ relacionado a $K$ en $t\left(\operatorname{con} \sum_{p \in k} w_{k t}=1\right)$.

Como segunda etapa, se multiplican las variables de tipo $X_{k i t}$ y $M_{k i t}$ por $w_{r t} / w_{k t}$ siendo $r$ un periodo perteneciente a $T$. Así, $X_{k i t}^{\prime}$ y $M_{k i t}^{\prime}$ indican los equivalentes ajustados de $X_{k i t}$ y $M_{\text {kit }}$ respectivamente:

$$
\left\{\begin{array}{l}
X_{k i t}^{\prime}=\frac{w_{r t}}{w_{k t}} X_{k i t} \\
M_{k i t}^{\prime}=\frac{w_{r t}}{w_{k t}} M_{k i t}
\end{array}\right.
$$

$r$ es un periodo de referencia en el cual se asume que los intercambios no se ven afectados por un sesgo coyuntural que limita su capacidad para revelar las ventajas/desventajas (o por lo menos el sesgo en cuestión es minimizado en $r$ ). Un sesgo coyuntural es una variación puntual en los intercambios, debido a por ejemplo un choque de oferta/ demanda, sin que esta variación deba reflejar un cambio en las ventajas/desventajas. 
Por tal razón, se asume que la ausencia de sesgo coyuntural se traduce por la estructura del comercio tal como lo dan a conocer las variables de tipo $w_{k r}$. Si por ejemplo $w_{k r}$ $=0,15$ entonces la ausencia de sesgo coyuntural se traduce por un comercio de $k$ que representa el $15 \%$ del comercio total. Si $w_{k t}=0,30 \mathrm{u}$ otro valor diferente a 0,15 el comercio de $k$ muestra entonces un sesgo coyuntural, por lo que es necesario ajustar las variables de tipo $X_{k i t}$ y $M_{k i t}$ para que resulten en un peso del $15 \%$ en lugar del $30 \%$, es decir, multiplicarlas por $1 / 2$. Este coeficiente de ajuste corresponde precisamente a $w_{r t} /$ $w_{k t}$ (en el ejemplo, $0,15 / 0,30$ ).

Como tercera etapa, se multiplica $w_{k t}$ por el saldo comercial total de $i$ en $t$, tomando las exportaciones y las importaciones ajustadas para calcular dicho saldo. En este sentido, $z_{\text {kit }}$ representa la variable subsecuente:

$$
z_{k i t}=w_{k t} \sum_{p \in K}\left(X_{p i t}^{\prime}-M_{p i t}^{\prime}\right)
$$

La variable $z_{k i t}$ es conceptualizada como el saldo comercial que $i$ debería registrar para que los intercambios revelen la ausencia tanto de ventajas comparativas como de desventajas comparativas en cuanto a $k$ en $t$. La hipótesis subyacente es la siguiente: la ausencia de ventajas/desventajas se traduce por una distribución del saldo comercial de un país a proporción del peso de cada producto en el comercio total de la zona considerada. En este contexto, como cuarta etapa, se calcula la diferencia entre: i) el saldo comercial efectivo (y ajustado), $X_{k i t}^{\prime}-M_{k i t}^{\prime} ;$ y ii) el saldo comercial teórico de (3). En esta línea de análisis, denota dicha diferencia:

$$
d_{k i t}=\left(X_{k i t}^{\prime}-M_{k i t}^{\prime}\right)-Z_{k i t}
$$

Si $d_{k i t}=0$ entonces ambos saldos son iguales, lo que implica la ausencia de ventajas/ desventajas. Por lo tanto, cero es el valor neutro del indicador VCR-CSC. Si $d_{k i t}>0$ entonces el saldo efectivo supera el saldo teórico, lo que implica la existencia de ventajas comparativas para $(k ; i ; t) ;$ y vice-versa si $d_{k i t}<0$.

Como etapa final, se expresa $d_{k i t}$ como una proporción de la producción nacional, a fin de tener en cuenta el tamaño económico de $i$ al revelar las ventajas/desventajas. Esto 
resulta en el indicador VCR-CSC, denotado $C S C_{\text {kit }}$ para el producto $k$, el país $i$ y el periodo t. Siendo GDP it el Producto Interno Bruto (PIB) de $i$ en $t$ :

$$
C S C_{k i t}=d_{k i t} / G D P_{i t}
$$

Bajo esta óptica, un PIB mayor resulta en un nivel menor de ventajas comparativas

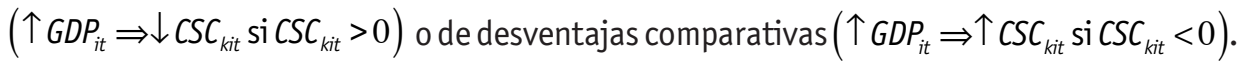

Combinando las ecuaciones (2) a (5), y siendo $w_{k t}=\sum_{i \in j}\left(X_{k i t}+M_{k i t}\right) / \sum_{i \in J} \sum_{p \in K}\left(X_{p i t}+M_{p i t}\right)$ tal como lo plantea la ecuación (1), el indicador VCR-CSC se escribe:

$$
C S C_{k i t}=\frac{1}{G D P_{i t}}\left[\frac{w_{r t}}{w_{k t}}\left(X_{k i t}-M_{k i t}\right)-w_{k t} \sum_{p \in K}\left(\frac{w_{r t}}{w_{p t}}\left(X_{p i t}+M_{p i t}\right)\right)\right]
$$

La tabla 1 da un ejemplo de cálculo del indicador VCR-CSC, para el producto denominado "Maíz (excepto maíz dulce), sin moler" (producto de la Clasificación Uniforme para el Comercio Internacional, identificado por el código 044), en el caso de Colombia frente a los otros países de la Alianza del Pacífico, en 2016.

\section{Tabla 1}

\section{Ilustración del cálculo del indicador VCR-CSC}

1. Cálculo de $w_{k t}$ :

1.1. $\sum_{i \in J} \sum_{p \in K}\left(X_{p i t}+M_{p i t}\right)=2,8983 e 10$ : según UNCTADStat, los miembros de la Alianza del Pacífico registraron en 2016 un comercio total de mil millones de dólares.

1.2. $\sum_{i \in J}\left(X_{k i t}+M_{k i t}\right)=2,8983 \mathrm{e} 10$ : los miembros de la Alianza del Pacífico registraron en 2016 un comercio de 28,644 millones de dólares respecto a la categoría de producto $k=$ [044], a saber, “Maíz (excepto maíz dulce), sin moler".

1.3. $w_{k t}=0,000988:[044]$ representa $0,0988 \%$ del comercio total de los miembros de la Alianza del Pacífico en 2016.

2. Cálculo de $w_{k r}$ :

2.1. $\sum_{i \in J} \sum_{p \in K}\left(X_{p i r}+M_{p i r}\right)=6,0482 \mathrm{e} 09$ : los miembros de la Alianza del Pacífico registró en el año de referencia $r=1995$ un comercio total de 6,0482 mil millones de dólares.

2.2. $\sum_{i \in J}\left(X_{k i r}+M_{k i r}\right)=4,7225 \mathrm{e} 05$ : los miembros de la Alianza del Pacífico registraron en 1995 un comercio de 472,25 miles de dólares respecto a la categoría de producto $k=$ [044].

2.3. $w_{k r}=0,000078$ : [044] representa $0,0078 \%$ del comercio total de los miembros de la Alianza del Pacífico en 1995. 
3. Cálculo de los flujos comerciales ajustados:

3.1. $\frac{w_{r t}}{w_{k t}}=\frac{0,000078}{0,000988}=0,07901$

3.2. $X_{k i t}^{\prime}=\frac{w_{r t}}{w_{k t}} X_{k i t}=0,07901 \times 1,2404 \mathrm{e} 05=9799,78$ : Las exportaciones de [044] desde Colombia hacia los otros miembros de la Alianza del Pacífico suman 124,04 miles de dólares en 2016, lo que se ajusta a 9799,78 dólares.

3.3. $M_{k i t}^{\prime}=\frac{w_{r t}}{w_{k t}} M_{k i t}=0,07901 \times 18212=1438,89:$ las importaciones de [044] por Colombia desde los otros miembros de la Alianza del Pacífico suman 18212 dólares en 2016, lo que se ajusta a 1438,89 miles de dólares.

4. Cálculo del saldo comercial teórico:

4.1. Ajustando las exportaciones e importaciones de todas las otras categorías de productos de la Clasificación Uniforme para el Comercio Internacional, tal como se hace en el punto 3, es posible calcular el saldo comercial total ajustado de Colombia: $\sum_{p \in K}\left(X_{p i t}^{\prime}-M_{p i t}^{\prime}\right)=-1,2341 \mathrm{e} 09$.

4.2. Por lo tanto, $z_{k i t}=w_{k t} \sum_{p \in K}\left(X_{p i t}^{\prime}-M_{p i t}^{\prime}\right)=9,8827 \mathrm{e}-04 \times-1,2341 \mathrm{e} 09=-1,2196 \mathrm{e} 06$. La ausencia de ventajas/desventajas en 2016 para Colombia en cuanto a la categoría de producto [044] se revela por un saldo comercial de -1,2196 millones de dólares.

5. Cálculos finales:

5.1. La diferencia entre el saldo efectivo y el saldo teórico es $d_{k i t}=\left(X_{k i t}^{\prime}-M_{k i t}^{\prime}\right)-z_{k i t}=9799,78-1438,89$ $-(-1,2196 \mathrm{e} 06)=1,2280 \mathrm{e} 06$.

5.2. El PIB de Colombia en 2016 siendo de $2,8246 \mathrm{e} 11 \$$, CSC $_{\text {kit }}=d_{\text {kit }} / 2,8246 \mathrm{e} 11=1,2280 \mathrm{e} 06 / 2,8246 \mathrm{e} 11$ $\approx 4,3473 \mathrm{e}-06$. El indicador es positivo y por lo tanto revela ventajas comparativas.

Fuente: elaboración propia.

Como se mencionó brevemente en la introducción, el indicador VCR-CSC no constituye la única manera de revelar ventajas/desventajas, pues existen otros indicadores VCR. Por lo tanto, se procede a justificar el uso del indicador VCR-CSC y a explicar las razones por las cuales no se utilizan otros indicadores VCR disponibles en la literatura para la realización de este artículo. La siguiente sección trata este punto.

\section{Justificación del uso del indicador VCR-CSC}

En esta sección se hace una comparación entre el indicador VCR-CSC y otros cuatro indicadores VCR representativos en la literatura:

1. El indicador VCR estándar (Balassa, 1965)

2. El indicador VCR "aditivo" de Hoen y Oosterhaven (2006) 
3. La versión "simétrica" del indicador estándar (Laursen, 2015)

4. El indicador VCR de Yu et ál. (2009)

Se empieza por presentar cada uno de estos indicadores, los cuales se basan únicamente en las exportaciones para la realización de los cálculos.

- El indicador VCR estándar (Balassa, 1965) toma como variable derivada de los intercambios el peso de $k$ en las exportaciones totales de $i$ en $t$, es decir, $X_{k i t} / \sum_{p \in K} X_{p i t}$. Esta variable no revela ni ventajas ni desventajas si es igual al mismo peso calculado para el conjunto de países pertenecientes a $J$, es decir, $\sum_{i \in J} X_{k i t} / \sum_{i \in J} \sum_{p \in K} X_{p i t}$. El indicador VCR estándar, denotado por $B$, se calcula dividendo el primer peso por el segundo:

$$
B_{k i t}\left(\frac{X_{k i t}}{\sum_{p \in K} X_{p i t}}\right) /\left(\frac{\sum_{i \in J} X_{k i t}}{\sum_{i \in J} \sum_{p \in K} X_{p i t}}\right)
$$

Por lo tanto, 1 es el valor neutro del indicador. Si $B_{k i t}>1$, el resultado revela ventajas comparativas. Por el contrario, si $B_{k i t} \in[0 ; 1)$, el resultado señala desventajas comparativas.

- Hoen y Oosterhaven (2006) también basan su indicador VCR en los pesos $X_{k i t} / \sum_{p \in K} X_{p i t}$ y $\sum_{i \in J} X_{k i t} / \sum_{i \in J} \sum_{p \in K} X_{p i t}$. No obstante, estos calculan la diferencia entre los dos pesos en lugar de dividir el primer peso por el segundo. Siendo $H$ este indicador VCR:

$$
H_{k i t}=\frac{X_{k i t}}{\sum_{p \in K} X_{p i t}}-\frac{\sum_{i \in J} X_{k i t}}{\sum_{i \in J} \sum_{p \in K} X_{p i t}}
$$

Cero es el valor neutro del indicador. Así, si $H_{k i t}>0$, este revela ventajas comparativas; y si $H_{k i t}<0$, se revelan por el contrario desventajas comparativas.

- Laursen (2015) sugiere una transformación del indicador VCR de Balassa (1965) que se aproxima a una aplicación del logaritmo a $B$. Siendo $L$ este indicador VCR:

$$
L_{k i t}=\left(B_{k i t}-1\right) /\left(B_{k i t}+1\right)
$$


Teniendo en cuenta que cero es el valor neutro del indicador, $L_{k i t} \in(0 ; 1]$ revela ventajas comparativas y $L_{k i t} \in[-1 ; 0)$ indica desventajas comparativas.

- El indicador VCR de Yu et ál. (2009) se construye según el mismo procedimiento que el indicador VCR-CSC. La principal diferencia radica en que el indicador VCR de Yu et ál. (2009) no se calcula con base en las exportaciones y las importaciones, sino únicamente en las exportaciones. En este sentido: i) en lugar de un saldo comercial teórico asociado a la ausencia de ventajas/desventajas, Yu et ál. (2009) calculan un nivel teórico de exportaciones asociado a dicha ausencia; ii) este nivel teórico se calcula ponderando las exportaciones totales de $i$ por el peso de $k$ en las exportaciones de $J$ (en $t$ ), en lugar de ponderar el saldo comercial total de $i$ por el peso de $k$ en el comercio de $J$ (en $c)$; iii) además, no se ajustan las exportaciones (ver ecuación 2); iv) finalmente, se expresa la diferencia entre las exportaciones efectivas y las exportaciones teóricas como una proporción de las exportaciones totales de $J$ en lugar del PIB de $i$ (en $t$ ). Siendo $Y$ el indicador VCR de Yu et ál. (2009):

$$
Y_{k i t}=\frac{1}{\sum_{p \in J} \sum_{j \in J} X_{p j t}}\left[X_{k i t}-\frac{\sum_{j \in J} X_{k j t}}{\sum_{p \in K} \sum_{j \in J} X_{p j t}} \sum_{p \in K} X_{p i t}\right]
$$

De manera general, el indicador VCR-CSC supera los indicadores antes señalados porque su cálculo se basa, además de las exportaciones, en las importaciones y en el PIB, y no únicamente en las exportaciones como es el caso de otros indicadores. Esto permite revelar las ventajas/desventajas de manera más consistente (Danna-Buitrago y Stellian, 2014; Danna-Buitrago et ál., 2017; Stellian y Danna-Buitrago, 2017). Sin embargo, también es necesario explicar las razones por las cuales se selecciona el indicador VCRCSC, en el caso particular de la presente investigación, en lugar de otros indicadores VCR disponibles en la literatura.

Para ello, se realiza una comparación entre los diferentes indicadores VCR según el criterio de la estacionaridad tendencial en el tiempo: un indicador VCR no debe tender a cambiar de manera significativa de un periodo a otro, debido a que las ventajas/desventajas tienden a cambiar solamente en horizontes de largo plazo (Lafay, 1987). En este orden de ideas, si un indicador VCR se caracteriza por alguna volatilidad en el tiempo, para un cierto país y un cierto producto, existe entonces un mayor riesgo de que esta volatilidad no se vincule enteramente a cambios en las ventajas/desventajas. En otros 
términos, la volatilidad impide que las ventajas/desventajas sean reveladas de manera correcta por los intercambios.

En consecuencia, se debe privilegiar para el desarrollo de la investigación un indicador VCR en lugar de otro, si este cumple en mayor medida con el criterio de estacionaridad tendencial en el tiempo. Precisamente, esta sección muestra que el indicador VCR-CSC presenta el mejor nivel de cumplimento del criterio en cuestión. Para ello, precisamos los parámetros ${ }^{3}$ según los cuales se calcula cada indicador VCR.

- Periodos $(t)$ : todos los años desde 1 hasta 2016.

- Nomenclatura de productos: las 255 categorías de productos según la Clasificación Uniforme para el Comercio Internacional (CUCI) a tres dígitos en su tercera revisión: ${ }^{4} k=$ [001] "Animales vivos", [011] "Carne de ganado bovino", [012] "0tras carnes y despojos comestibles de carnes", y así sucesivamente hasta [971] “Oro no monetario (excepto minerales y concentrados de oro)", pasando por ejemplo por [572] "Polímeros de estireno, en formas primarias" o [678] "Alambre de hierro o acero".

- Conforme a la investigación planteada, $i=$ "Colombia" en el marco de la zona $J=$ $\{\mathrm{CL} ; \mathrm{CO} ; \mathrm{MX} ; \mathrm{PE}\}$ y luego la zona $\mathrm{J}=\{\mathrm{AU} ; \mathrm{CA} ; \mathrm{CL} ; \mathrm{CO} ; \mathrm{MX} ; \mathrm{NZ} ; \mathrm{PE} ; \mathrm{SG}\}$.

Se toman los datos sobre las exportaciones y las importaciones de UNCTADStat. ${ }^{5}$ Para el indicador VCR-CSC, los datos del PIB colombiano se toman del Banco Mundial ${ }^{6}$ y se fija 1995 como el periodo $r$ de referencia para ajustar las exportaciones y las importaciones (ver Eq. 2). La autora pone a la disposición de los lectores un archivo Excel que contienen todos los cálculos.

Para mostrar la superioridad del indicador VCR-CSC, se define la manera de medir la estacionaridad tendencial en el tiempo de un indicador VCR. La medición usual se basa en el cálculo de desviaciones estándares (Leromain y Orefice, 2014). Para un cierto país y un cierto producto, es posible calcular la desviación estándar de la serie de indicadores VCR

\footnotetext{
3 El ejemplo de la tabla 1 ya había sido desarrollado según estos parámetros.

4 unstats.un.org/unsd/cr/registry/regcst.asp?Cl=14\&Lg=3.

5 unctadstat.unctad.org/wds/ReportFolders/reportFolders.aspx.

6 data.worldbank.org/indicator/NY.GDP.MKTP.CD.
} 
desde el primer período hasta el último período en T. Esto da cuenta de la volatilidad del indicador alrededor de su promedio en el tiempo para el país considerado y el producto considerado (y una vez fijadas la zona a la cual pertenece el país y la nomenclatura a la que pertenece el producto). Una desviación estándar mayor implica una volatilidad mayor, lo que va en contra del criterio de estacionaridad tendencial en el tiempo. En este sentido, un indicador VCR se puede considerar como óptimo si tiende a minimizar las desviaciones estándares arriba mencionadas.

Así, a cada forma de calcular un indicador VCR (CSC, B, H, L e Y) y cada zona se vincula una matriz de 255 filas - una por categoría de mercancía- y 22 columnas -una por añoen el caso de Colombia; es decir, 10 matrices; por lo que cada una corresponde a una forma entre 5 formas y a una zona entre 2. Dentro de cada matriz, se encuentran 22 indicadores VCR por categoría de mercancía, uno por año, y se calcula precisamente la desviación estándar de cada indicador VCR a lo largo de los 22 años para cada una de las 255 categorías.

La tabla 2 muestra cómo se distribuyen las desviaciones estándares para cada forma y cada zona, a través de los diferentes deciles. Se puede constatar que el decil correspondiente al indicador VCR-CSC siempre es menor que el decil de mismo rango de los otros indicadores, tanto en el caso de la zona conformada por la Alianza del Pacífico como de la zona denominada Alianza del Pacífico+4. En consecuencia, el indicador VCR-CSC muestra la mayor concentración de desviaciones estándares hacia cero, lo que conlleva a elegir el indicador VCR-CSC para cumplir con el criterio de estacionaridad tendencial en el tiempo. Desde este punto de vista, el indicador VCR second-best es Y, seguido por $H$, luego $L$ y por ultimo $B$. De un indicador VCR al siguiente, un número cada vez mayor de desviaciones estándares se alejan de cero, y por lo tanto se cumple menos con el criterio de estacionaridad tendencial en el tiempo del indicador VCR.

\section{Tabla 2}

Distribución de las desviaciones estándares en el tiempo de los indicadores VCR de Colombia, 1995-2016, Alianza del Pacífico y Alianza del Pacífico+4

\begin{tabular}{cccccc}
\hline \multicolumn{5}{c}{ Alianza del Pacífico } \\
\hline Decile & CSC & $B$ & $H$ & $L$ & $Y$ \\
\hline 1 & $2,93 \mathrm{e}-06$ & 0,014008 & 0,00011 & 0,02588 & $4,82 \mathrm{e}-06$ \\
2 & $5,88 \mathrm{e}-06$ & 0,045814 & 0,000242 & 0,062995 & $1,12 \mathrm{e}-05$ \\
3 & $8,83 \mathrm{e}-06$ & 0,081975 & 0,0005 & 0,098563 & $1,9 \mathrm{e}-05$ \\
4 & $1,47 \mathrm{e}-05$ & 0,208308 & 0,000739 & 0,132603 & $3,12 \mathrm{e}-05$ \\
\hline & & & & & Continúa $\rightarrow$
\end{tabular}




\begin{tabular}{cccccc}
\hline 5 & $2,25 \mathrm{e}-05$ & 0,304729 & 0,001015 & 0,174507 & $4,14 \mathrm{e}-05$ \\
6 & $3,04 \mathrm{e}-05$ & 0,471789 & 0,001384 & 0,233155 & $5,52 \mathrm{e}-05$ \\
7 & $4,46 \mathrm{e}-05$ & 0,753185 & 0,001978 & 0,285749 & $7,95 \mathrm{e}-05$ \\
8 & $6,95 \mathrm{e}-05$ & 1,197852 & 0,002804 & 0,344863 & 0,000113 \\
9 & 0,000124 & 2,063805 & 0,005425 & 0,437042 & 0,000213 \\
10 & 0,000928 & 7,273624 & 0,105448 & 0,787406 & 0,004873 \\
\hline \multicolumn{5}{c}{ Alianza del Pacífico+4 } \\
\hline Decile & CSC & $B$ & $H$ & $L$ & $Y$ \\
\hline 1 & $7,25 \mathrm{e}-07$ & 0,039871 & $7,07 \mathrm{e}-05$ & 0,066773 & $1,5 \mathrm{e}-05$ \\
2 & $2,04 \mathrm{e}-06$ & 0,140935 & 0,000214 & 0,120762 & $4,38 \mathrm{e}-05$ \\
3 & $4,34 \mathrm{e}-06$ & 0,243794 & 0,000463 & 0,16093 & $9,31 \mathrm{e}-05$ \\
4 & $8,43 \mathrm{e}-06$ & 0,364209 & 0,00073 & 0,205543 & 0,00014 \\
5 & $1,31 \mathrm{e}-05$ & 0,5104 & 0,001072 & 0,240913 & 0,000198 \\
6 & $1,85 \mathrm{e}-05$ & 0,692794 & 0,001434 & 0,286953 & 0,000289 \\
7 & $2,75 \mathrm{e}-05$ & 0,851265 & 0,00205 & 0,339478 & 0,000371 \\
8 & $4,54 \mathrm{e}-05$ & 1,021587 & 0,003177 & 0,420608 & 0,000599 \\
9 & $9,82 \mathrm{e}-05$ & 1,399222 & 0,006071 & 0,547427 & 0,001207 \\
10 & 0,001591 & 2,94866 & 0,095844 & 0,775741 & 0,02179 \\
\hline
\end{tabular}

Fuente: elaboración propia.

Se implementa una medición adicional de la estacionaridad tendencial en el tiempo, con base en la siguiente regresión lineal. Siendo $V C R_{k i t}$ un indicador VCR calculado para $(k ; i ; t), t_{0}$ un periodo inicial $t_{1}>t_{0}$ un periodo final (Laursen, 2015):

$$
V C R_{k i t_{1}}=\alpha+\beta \cdot V C R_{k i t_{0}}+\varepsilon_{i k}
$$

Un indicador VCR de $i$ en $t_{1}$ es conceptualizado como la variable dependiente, y el mismo indicador en $t_{0}$ es conceptualizado como la variable independiente. Así se considera el conjunto de indicadores calculados para cada $k \in K$ como una observación del indicador tanto en $t_{0}$ como en $t_{1}$ (por ende, son 255 observaciones con la nomenclatura CUCI). Bajo esta óptica, la estacionaridad tendencial entre $t_{0}$ y $t_{1}$ se traduce por $\alpha \rightarrow 0$ y $\beta \rightarrow 1$, pues en este caso un indicador VCR en $t_{1}$ es diferente del indicador correspondiente en $t_{0}$ solo por el término de error $\varepsilon_{i k^{*}}$. La tabla 3 da cuenta de las regresiones, tomando 1995 como el periodo $t_{0}$ (primer año disponible) y 2016 como periodo $t_{1}$ (último año disponible), y se utilizan los indicadores VCR calculados basándose en la zona Alianza del Pacífico, antes de hacer lo mismo con la zona Alianza del Pacífico+4. 
En el caso de la zona Alianza del Pacífico, los indicadores CSC, $H$ e Yimplican $\alpha \rightarrow 0$, tal como lo dan a conocer la estimación de $\alpha$, así como la $t$-value correspondiente y el intervalo de confianza de $95 \%$. Luego, el indicador CSC permite que el coeficiente $\beta$ se sitúe lo más cerca de $1: 1,3752$, es decir, una distancia de 0,3752 respecto a 1 , contra una distancia de $|0,1953-1|=0,8147$ para el indicador $H$ y $|0,1361-1|=0,9639$ para el indicador $Y$.

\section{Tabla 3}

Estimación de la Ecuación 12, Colombia, $t_{0}=1995 y t_{1}=2016$, Alianza del Pacífico y Alianza del Pacífico+4

\begin{tabular}{|c|c|c|c|c|c|c|c|}
\hline \multicolumn{8}{|c|}{ Alianza del Pacífico } \\
\hline Indicador VCR & \multicolumn{2}{|c|}{ Estimación } & \multirow{2}{*}{$\begin{array}{l}\text { Des. est. } \\
0,0000\end{array}$} & \multirow{2}{*}{$\begin{array}{l}\text {-value } \\
0,0000\end{array}$} & \multirow{2}{*}{$\begin{array}{l}P(>|t|) \\
1,0000\end{array}$} & \multicolumn{2}{|c|}{ Intervalo de confianza (95\%) } \\
\hline \multirow{2}{*}{$\csc$} & $\alpha$ & 0,0000 & & & & $-0,0000161$ & 0,0000161 \\
\hline & $\beta$ & 1,3752 & 0,0405 & 33,9806 & 0,0000 & 1,295506 & 1,454909 \\
\hline \multirow{2}{*}{ B } & $\alpha$ & 0,5366 & 0,0857 & 6,2602 & 0,0000 & 0,3678244 & 0,7054726 \\
\hline & $\beta$ & 0,5919 & 0,0682 & 8,6742 & 0,0000 & 0,4575426 & 0,7263288 \\
\hline \multirow{2}{*}{ H } & $\alpha$ & $-0,0000$ & 0,0005 & $-0,0000$ & 1,0000 & $-0,0010813$ & 0,0010813 \\
\hline & $\beta$ & 0,1953 & 0,0325 & 6,0168 & 0,0000 & 0,131353 & 0,2591787 \\
\hline \multirow{2}{*}{ L } & $\alpha$ & $-0,0856$ & 0,0368 & $-2,3258$ & 0,0208 & $-0,1580383$ & $-0,0131132$ \\
\hline & $\beta$ & 0,5244 & 0,0540 & 9,7094 & 0,0000 & 0,4180138 & 0,630735 \\
\hline \multirow{2}{*}{$Y$} & $\alpha$ & $-0,0000$ & 0,0001 & $-0,0000$ & 1,0000 & $-0,0001981$ & 0,0001981 \\
\hline & $\beta$ & 0,1361 & 0,0226 & 6,0168 & 0,0000 & 0,0915489 & 0,1806395 \\
\hline \multicolumn{8}{|c|}{ Alianza del Pacífico+4 } \\
\hline Indicador VCR & \multicolumn{2}{|c|}{ Estimación } & Des. est. & $t$-value & $P(>|t|)$ & \multicolumn{2}{|c|}{ Intervalo de confianza ( $95 \%)$} \\
\hline \multirow{2}{*}{ CSC } & $\alpha$ & $-0,0000$ & 0,0000 & $-0,0000$ & 1,0000 & $-0,0000169$ & 0,0000169 \\
\hline & $\beta$ & 0,4554 & 0,0352 & 12,9417 & 0,0000 & 0,3860919 & 0,5246886 \\
\hline \multirow{2}{*}{ B } & $\alpha$ & 0,5683 & 0,1570 & 3,6204 & 0,0004 & 0,259147 & 0,8773909 \\
\hline & $\beta$ & 0,7417 & 0,0589 & 12,5911 & 0,0000 & 0,6256807 & 0,8576967 \\
\hline \multirow{2}{*}{$\mathrm{H}$} & $\alpha$ & 0,0000 & 0,0007 & 0,0000 & 1,0000 & $-0,0012952$ & 0,0012952 \\
\hline & $\beta$ & 0,2139 & 0,0331 & 6,4688 & 0,0000 & 0,148768 & 0,2789985 \\
\hline \multirow{2}{*}{ L } & $\alpha$ & $-0,0238$ & 0,0359 & $-0,6626$ & 0,5082 & $-0,0943869$ & 0,0468632 \\
\hline & $\beta$ & 0,6872 & 0,0463 & 14,8313 & 0,0000 & 0,5959432 & 0,7784419 \\
\hline \multirow{2}{*}{ Y } & $\alpha$ & 0,0000 & 0,0000 & 0,0000 & 1,0000 & $-0,0000571$ & 0,0000571 \\
\hline & $\beta$ & 0,2196 & 0,0339 & 6,4688 & 0,0000 & 0,1527517 & 0,2864695 \\
\hline
\end{tabular}

Fuente: elaboración propia. 
Además, lo mismo se puede verificar en el caso de la zona Alianza del Pacífico+4: se seleccionan CSC, $H$ e $Y$ porque implican $\alpha \rightarrow 0$, antes de seleccionar CSC, ya que además minimiza $|\beta-1|$. En consecuencia, entre los diferentes indicadores estudiados, el indicador CSC se acerca más de las condiciones $\alpha \rightarrow 0$ y $\beta \rightarrow 1$ de tal manera que este indicador cumple en mayor medida con el criterio de estacionaridad tendencial en el tiempo así medido, para las dos zonas consideradas de intercambio.

Como el indicador VCR-CSC es el más consistente según el criterio de estacionaridad tendencial en el tiempo de acuerdo con el conjunto de pruebas antes efectuadas, este se elige para la realización de la investigación. Así pues, se procede al análisis de las posibles influencias de la Alianza del Pacífcio+4 sobre la especialización regional de Colombia a partir del indicador VCR-CSC. Se espera que las pruebas antes realizadas, para verificar el criterio de estacionaridad tendencial en el tiempo, sustenten la utilización del indicador VCR-CSC para otras investigaciones, en donde se involucren otros países y otras zonas de intercambios.

\section{Análisis del indicador VCR-CSC: influencia de la Alianza del Pacífico+4 sobre la especialización regional de Colombia}

La tabla 4 contiene estadísticas descriptivas del indicador VCR-CSC para cada año. Con el fin de analizar las implicaciones de la Alianza del Pacífico+4 sobre la especialización regional de Colombia, se calcula el promedio en el tiempo de CSC $_{k i t}$ para cada categoría $k$ de productos. El promedio se calcula según un factor $\mu$ de suavización exponencial, para dar más peso a los indicadores de los últimos años. Tomando 1995 como el año 1 , 1996 como el año 2, y así sucesivamente hasta 2016 como el año 22:

$$
\overline{\operatorname{CSC}}_{k i}=\frac{\sum_{t=1}^{22}(1-\mu)^{22-t} \operatorname{CSC}_{k i t}}{\sum_{t=1}^{22}(1-\mu)^{22-t}}
$$

Se utiliza $\mu=0,15$, de tal manera que se pondera CSC $_{k i 22}$ por 1 (año 2016) al calcular $\overline{C S C}_{k i}$, luego el coeficiente ponderando CSC $_{k i 21}$ es 0,85 (año 2015), antes de 0,7225 para CSC $_{\text {ki2o }}$ (año 2014), y así sucesivamente hasta 0,03295 para CSC ${ }_{k i 1}$ (año 1995). Denotamos $\overline{C S C}_{k i}^{1}$ la variable $\overline{\mathrm{CSC}}_{k i}$ calculada en el marco de la zona $\mathrm{J}=\{\mathrm{CL} ; \mathrm{CO} ; \mathrm{MX} ; \mathrm{PE}\}$ y $\overline{\mathrm{CSC}}_{k i}^{2}$ la variable $\overline{\mathrm{CSC}}_{k i}$ calculada en el marco de la zona $\mathrm{J}=\{\mathrm{AU} ; \mathrm{CA} ; \mathrm{CL} ; \mathrm{CO} ; \mathrm{MX} ; \mathrm{NZ} ; \mathrm{PE} ; \mathrm{SG}\}$. 
Tabla 4

Indicador VCR-CSC: estadísticas descriptivas

\begin{tabular}{|c|c|c|c|c|c|c|c|c|}
\hline & \multicolumn{4}{|c|}{ Colombia en la Alianza del Pacífico } & \multicolumn{4}{|c|}{ Colombia en la Alianza del Pacífico+4 } \\
\hline & Media & Desv. est. & Min & Max & Media & Desv. est. & Min & $\operatorname{Max}$ \\
\hline 1995 & $1,42 e-20$ & $2,02 e-04$ & $-9,81 e-04$ & $2,74 \mathrm{e}-03$ & $-5,16 e-21$ & $2,44 \mathrm{e}-04$ & $-9,58 e-04$ & $3,20 e-03$ \\
\hline 1996 & $3,32 \mathrm{e}-22$ & $2,09 e-04$ & $-9,35 e-04$ & $2,86 e-03$ & $-1,38 \mathrm{e}-21$ & $2,21 \mathrm{e}-04$ & $-1,09 e-03$ & $2,54 \mathrm{e}-03$ \\
\hline 1997 & $3,82 e-21$ & $2,17 e-04$ & $-8,11 e-04$ & $3,07 e-03$ & $-1,06 e-22$ & $1,81 \mathrm{e}-04$ & $-8,04 e-04$ & $1,93 e-03$ \\
\hline 1998 & $3,03 e-21$ & $2,13 e-04$ & $-7,60 e-04$ & $3,08 \mathrm{e}-03$ & $-3,51 e-21$ & $1,59 \mathrm{e}-04$ & $-6,57 e-04$ & $1,64 \mathrm{e}-03$ \\
\hline 1999 & $-5,05 e-22$ & $2,08 e-04$ & $-7,85 e-04$ & $2,94 \mathrm{e}-03$ & $-1,70 e-21$ & $1,68 \mathrm{e}-04$ & $-6,92 e-04$ & $1,57 e-03$ \\
\hline 2000 & $4,19 e-21$ & $1,91 \mathrm{e}-04$ & $-1,06 \mathrm{e}-03$ & $2,53 e-03$ & $-5,31 e-22$ & $1,52 \mathrm{e}-04$ & $-9,29 e-04$ & $1,14 \mathrm{e}-03$ \\
\hline 2001 & $-1,01 \mathrm{e}-21$ & $1,13 e-04$ & $-1,15 e-03$ & $5,19 e-04$ & $-5,31 e-21$ & $1,49 e-04$ & $-9,53 e-04$ & $1,11 \mathrm{e}-03$ \\
\hline 2002 & $-2,39 \mathrm{e}-22$ & $1,20 \mathrm{e}-04$ & $-9,55 e-04$ & $7,91 \mathrm{e}-04$ & $-2,60 e-21$ & $1,55 \mathrm{e}-04$ & $-7,66 e-04$ & $1,16 \mathrm{e}-03$ \\
\hline 2003 & $-1,57 e-21$ & $1,42 \mathrm{e}-04$ & $-1,18 \mathrm{e}-03$ & $8,19 e-04$ & $-2,50 \mathrm{e}-21$ & $1,77 e-04$ & $-9,79 e-04$ & $1,33 e-03$ \\
\hline 2004 & $-1,64 \mathrm{e}-21$ & $1,95 e-04$ & $-1,40 e-03$ & $1,84 \mathrm{e}-03$ & $1,89 \mathrm{e}-21$ & $1,73 e-04$ & $-8,83 e-04$ & $1,34 \mathrm{e}-03$ \\
\hline 2005 & $2,31 \mathrm{e}-22$ & $2,68 \mathrm{e}-04$ & $-1,47 e-03$ & $3,29 \mathrm{e}-03$ & $-7,55 e-21$ & $1,94 \mathrm{e}-04$ & $-9,52 e-04$ & $1,39 \mathrm{e}-03$ \\
\hline 2006 & $-8,79 e-21$ & $2,39 \mathrm{e}-04$ & $-1,82 \mathrm{e}-03$ & $1,19 e-03$ & $-1,68 \mathrm{e}-20$ & $2,26 e-04$ & $-1,38 \mathrm{e}-03$ & $1,39 \mathrm{e}-03$ \\
\hline 2007 & $-9,23 e-22$ & $2,02 e-04$ & $-1,37 e-03$ & $1,07 e-03$ & 1,11e-20 & $2,30 \mathrm{e}-04$ & $-1,72 \mathrm{e}-03$ & $1,39 \mathrm{e}-03$ \\
\hline 2008 & $-1,24 \mathrm{e}-20$ & $2,62 e-04$ & $-1,46 e-03$ & $3,13 e-03$ & $-1,28 e-20$ & $2,40 \mathrm{e}-04$ & $-1,45 e-03$ & $1,72 \mathrm{e}-03$ \\
\hline 2009 & $-2,66 e-23$ & $1,34 \mathrm{e}-04$ & $-1,20 \mathrm{e}-03$ & $4,78 \mathrm{e}-04$ & $-6,80 e-21$ & $1,92 \mathrm{e}-04$ & $-1,30 e-03$ & $1,12 \mathrm{e}-03$ \\
\hline 2010 & $7,14 \mathrm{e}-22$ & $2,71 \mathrm{e}-04$ & $-1,56 e-03$ & $3,52 \mathrm{e}-03$ & $1,02 \mathrm{e}-20$ & $2,54 \mathrm{e}-04$ & $-2,08 e-03$ & $1,88 \mathrm{e}-03$ \\
\hline 2011 & $9,80 \mathrm{e}-21$ & $3,84 \mathrm{e}-04$ & $-2,03 e-03$ & $5,34 \mathrm{e}-03$ & $-1,53 \mathrm{e}-20$ & $3,55 e-04$ & $-3,55 e-03$ & $3,10 \mathrm{e}-03$ \\
\hline 2012 & $1,66 \mathrm{e}-20$ & $3,27 e-04$ & $-9,34 \mathrm{e}-04$ & $4,65 e-03$ & $9,78 \mathrm{e}-21$ & $2,61 \mathrm{e}-04$ & $-1,87 e-03$ & $2,80 \mathrm{e}-03$ \\
\hline 2013 & $-2,75 e-21$ & $2,70 e-04$ & $-7,63 e-04$ & $3,82 \mathrm{e}-03$ & $-1,49 e-20$ & $1,87 e-04$ & $-9,91 e-04$ & $1,53 \mathrm{e}-03$ \\
\hline 2014 & $-4,63 e-21$ & $2,29 \mathrm{e}-04$ & $-7,81 \mathrm{e}-04$ & $2,94 \mathrm{e}-03$ & $-1,11 \mathrm{e}-20$ & $2,16 e-04$ & $-1,67 e-03$ & $1,35 \mathrm{e}-03$ \\
\hline 2015 & $-6,16 e-21$ & $3,33 e-04$ & $-1,16 e-03$ & $4,76 e-03$ & $0,00 \mathrm{e}+00$ & $2,06 e-04$ & $-1,30 e-03$ & $1,89 \mathrm{e}-03$ \\
\hline 2016 & $-3,16 e-22$ & $3,07 e-04$ & $-1,03 e-03$ & $4,40 e-03$ & $1,26 \mathrm{e}-20$ & $1,76 \mathrm{e}-04$ & $-1,45 e-03$ & $1,11 \mathrm{e}-03$ \\
\hline
\end{tabular}

Fuente: elaboración propia.

Nota: la media tiende hacia cero en cada año. Esto resulta de la construcción del indicador VCR-CSC. En efecto, la sumatoria de los indicadores calculados para las diferentes categorías de productos tiende hacia cero (De Saint Vaulry, 2008). Por lo tanto, la división de la sumatoria por el número de categorías, de donde resulta la media, también tiende hacia cero.

Tomando como referente lo antes señalado, es posible identificar cuatro cambios principales pasando de $\overline{\operatorname{CSC}}_{k i}^{1}$ a $\overline{\operatorname{CSC}}_{k 0}^{2}$. De acuerdo con lo expresado, desde la zona Alianza del Pacífico hacia la zona Alianza del Pacífico+4, los intercambios de Colombia respecto a la categoría de producto... 
1. ... revelan ventajas comparativas en lugar de desventajas comparativas si $\overline{C S C}_{k i}^{1}<$ 0 y $\overline{C S C}_{k i}^{2}>0$. En consecuencia, k sería un punto fuerte de Colombia en la liberalización del comercio hacia los nuevos miembros de la Alianza del Pacífico.

2. ... siguen revelando ventajas comparativas $\mathrm{si} \overline{\operatorname{CSC}}_{k i}^{1}>0$ y $\overline{\operatorname{CSC}}_{k i}^{2}>0$. Solo se cambia el grado de estas ventajas, las cuales son menores si $\overline{\operatorname{CSC}}_{k i}^{1}>\overline{\operatorname{CSC}}_{k^{\prime}}^{2}$ y vice-versa.

3. ... siguen revelando desventajas comparativas $\operatorname{si} \overline{\operatorname{CSC}}_{b i}^{1}<0$ y $\overline{\operatorname{CSC}}_{b i}^{2}<0$. Solo se cambia el grado de estas desventajas, las cuales son menores si $\overline{\operatorname{CSC}}_{k i}^{1}<\overline{\operatorname{CSC}}_{k i}^{2}$ y vice-versa.

4. ... revelan desventajas comparativas en lugar de ventajas comparativas $\operatorname{si}_{\mathrm{CSC}_{k i}}^{1}>0$ $y \overline{C S C}_{k i}^{2}<0$. En consecuencia, $k$ sería un punto débil de Colombia en la liberalización del comercio hacia los nuevos miembros de la Alianza del Pacífico.

La gráfica 1 muestra cómo las 255 categorías k estudiadas se distribuyen entre los cuatro cambios, situándose $\overline{\operatorname{CSC}}_{k i}^{1}$ en el eje horizontal y $\overline{\operatorname{CSC}}_{k i}^{2}$ en el eje vertical. Así, un punto en el cuadrante 1 corresponde a una categoría asociada al primer cambio, es decir, $\overline{\operatorname{CSC}}_{k i}^{1}<$ 0 y $\overline{C S C}_{k i}^{2}>0$; un punto en el cuadrante 2 corresponde a una categoría asociada al segundo cambio, es decir, $\overline{\operatorname{CSC}}_{k i}^{1}>0$ y $\overline{\operatorname{CSC}}_{k i}^{2}>0$; e igual para los cuadrantes 3 y 4 . La gráfica 1 muestra que la mayor parte de las categorías se concentran en los cuadrantes 2 y 3 . En consecuencia, la mayor parte de categorías se relacionan a los cambios 2 y 3 , es decir, conservan sus ventajas (cambio 2: $\overline{\mathrm{CSC}}_{k i}^{1}>0 \mathrm{y} \overline{\mathrm{CSC}}_{k i}^{2}>0$ ) o conservan sus desventajas (cambio 3: $\overline{\operatorname{CSC}}_{k i}^{1}<0$ y $\overline{\operatorname{CSC}}_{k i}^{2}<0$ ) pasando de la Alianza del Pacífico a la Alianza del Pacífico+4. Para estas categorías, el esquema de especialización de Colombia no se vería afectado por la conformación de la Alianza del Pacífico+4.

En la tabla 5 se evidencia que 116 categorías se asocian al cambio 2 (conservan sus ventajas) y 83 categorías se asocian al cambio 3 (conservan sus desventajas). En otros términos, los cambios 2 y 3 constituyen más del $75 \%$ del total de las categorías, siendo 255 el total. En cuanto a los otros dos cambios, es decir, el cambio 1 (obtención de ventajas) y el cambio 4 (perdida de ventajas), la tabla 5 muestra que muchas más categorías pierden sus ventajas al pasar de la Alianza del Pacífico a la Alianza del Pacífico+4, en comparación al número de categorías que obtienen ventajas desde una zona hacia la siguiente: 32 versus 24. En consecuencia, se puede conjeturar que la liberalización del comercio desde la Alianza del Pacífico hacia Australia, Canadá, Nueva Zelandia y Singapur crearía más amenazas que oportunidades para la especialización regional de Colombia. 


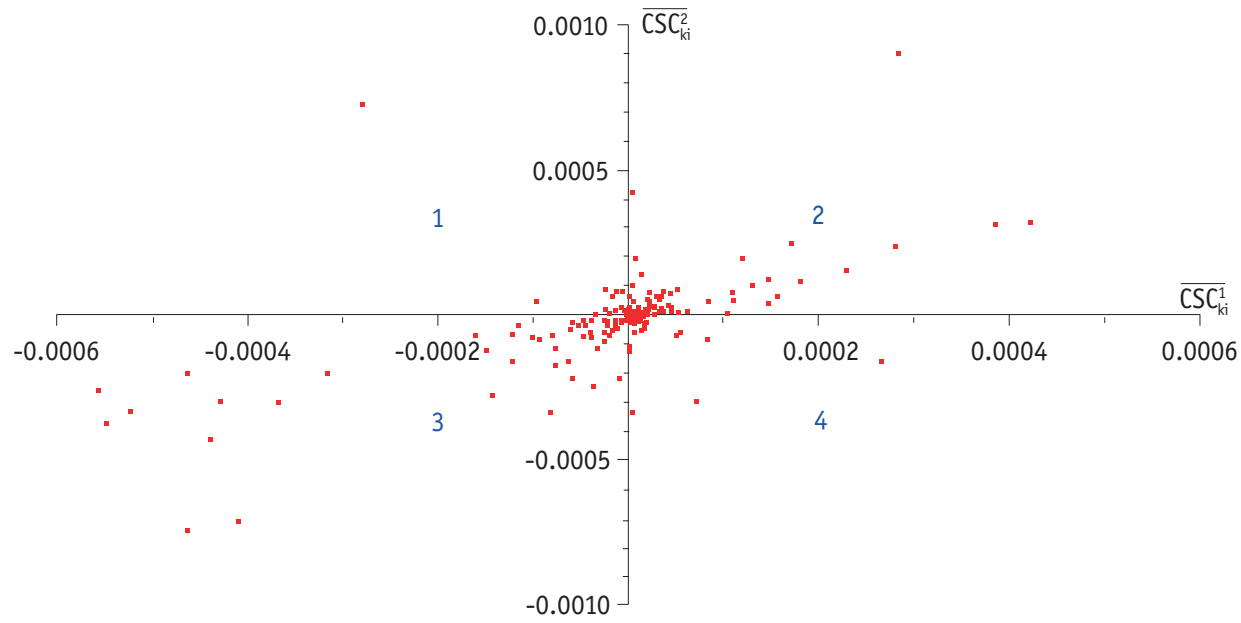

\section{Gráfica 1. Conjunto de puntos $\left(\overline{\operatorname{CSC}}_{k i}^{1} ; \overline{\operatorname{CSC}}_{k i}^{2}\right)$}

Fuente: elaboración propia.

Nota: para el cálculo de $\overline{C S C}_{k i}^{1}$ y $\overline{C S C}_{k i^{\prime}}^{2}$ ver Eq. 7 y el párrafo relacionado. Los números 1 a 4 identifican los diferentes cuadrantes y los cuatro cambios correspondientes.

Igualmente, la tabla 5 provee detalles sobre la naturaleza de los productos asociados a cada uno de los cuatro cambios, con base en los diez principales grupos o capítulos de la CUCI, los cuales corresponden al primero de los tres dígitos que identifican cada una de las 255 categorías CUCI (ver tabla 6). Al respecto, se puede constatar que los artículos manufacturados clasificados principalmente según el material (capítulo 6) y la maquinaria y equipo de transporte (capítulo 7) concentran la mitad de las categorías asociadas a los cambios 1 y 4 :

- 12 de las 24 categorías asociadas al cambio $1\left(\overline{\operatorname{CSC}}_{k i}^{1}<0\right.$ y $\left.\overline{\operatorname{CSC}}_{k i}^{2}>0\right)$. De estas 12 categorías, 4 pertenecen al capítulo 6 y las 8 otras categorías pertenecen al capítulo 7.

- 16 de las 32 categorías asociadas al cambio $4\left(\overline{\operatorname{CSC}}_{k i}^{1}>0\right.$ y $\left.\overline{\operatorname{CSC}}_{k i}^{2}<0\right)$. De estas 16 categorías, 7 pertenecen al capítulo 6 y las otras 9 categorías pertenecen al capítulo 7. 


\section{Tabla 5}

Distribución de las 255 categorías CUCI a tres dígitos entre los diferentes capítulos $\mathrm{CUCl}$ y los diferentes cambios en las ventajas/desventajas de Colombia con la conformación de la Alianza del Pacífico+4

\begin{tabular}{|c|c|c|c|c|c|}
\hline \multirow{4}{*}{ Capítulo CUCI } & \multicolumn{4}{|c|}{ Cambio de las ventajas/desventajas comparativas } & \multirow{4}{*}{ Total } \\
\hline & 1 & 2 & 3 & 4 & \\
\hline & $\overline{\operatorname{CSC}}_{k i}^{1}<0$ & $\overline{\operatorname{CSC}}_{k i}^{1}>0$ & $\overline{\operatorname{CSC}}_{k i}^{1}<0$ & $\overline{\operatorname{CSC}}_{k i}^{1}>0$ & \\
\hline & $\overline{\operatorname{CSC}}_{k i}^{2}>0$ & $\overline{\operatorname{CSC}}_{k i}^{2}>0$ & $\overline{\operatorname{CSC}}_{k i}^{2}<0$ & $\overline{\operatorname{CSC}}_{k i}^{2}<0$ & \\
\hline 0 & 1 & 14 & 13 & 8 & 36 \\
\hline 1 & 1 & 3 & 0 & 0 & 4 \\
\hline 2 & 3 & 19 & 17 & 4 & 34 \\
\hline 3 & 2 & 7 & 0 & 1 & 10 \\
\hline 4 & 1 & 2 & 1 & 0 & 4 \\
\hline 5 & 2 & 15 & 14 & 2 & 33 \\
\hline 6 & 4 & 19 & 22 & 7 & 52 \\
\hline 7 & 8 & 14 & 20 * & 9 & 50 \\
\hline 8 & 2 & 22 & 6 & 1 & 31 \\
\hline 9 & 0 & 1 & 0 & 0 & 1 \\
\hline \multirow{2}{*}{ Total } & 24 & 116 & 83 & 32 & 255 \\
\hline & $9,41 \%$ & $45,49 \%$ & $32,16 \%$ & $12,55 \%$ & $100 \%$ \\
\hline
\end{tabular}

Fuente: elaboración propia.

Nota: para el cálculo de $\overline{C S C}_{k i}^{1}$ y $\overline{C S C}_{k{ }^{\prime}}^{2}$ véanse Eq. 7 y el párrafo relacionado. Para la descripción de cada capítulo CUCI, véase tabla 4. El asterisco señala que $\overline{C S C}_{k i}^{1}=0$ en el caso de una categoría de producto (286 - Minerales y concentrado de uranio o torio), es decir, $\overline{\operatorname{CSC}}_{k i}^{1} \leq 0$ en lugar de $\overline{\operatorname{CSC}}_{k i}^{1}<0$.

Tabla 6

Capítulos de la Clasificación Uniforme para el Comercio Internacional

\begin{tabular}{cl}
\hline Capítulo & \\
\hline 0 & Productos alimenticios y animales vivos \\
1 & Bebidas y tabacos \\
2 & Materiales crudos no comestibles (excepto los combustibles) \\
3 & Combustibles, lubricantes minerales y productos conexos \\
4 & Aceites, grasas y ceras de origen animal y vegetal \\
5 & Productos químicos y productos conexos, n.e.p. \\
\hline
\end{tabular}




\begin{tabular}{cl}
\hline Capítulo & \multicolumn{1}{c}{ Descripción } \\
\hline 6 & Artículos manufacturados, clasificados principalmente según el material \\
7 & Maquinaria y equipo de transporte \\
8 & Artículos manufacturados diversos \\
9 & Oro no monetario (excepto minerales y concentrados de oro) \\
\hline
\end{tabular}

Fuente: unstats.un.org/unsd/cr/registry/regcst.asp?Cl=14\&Lg=3\&Top=1.

Nota: el capítulo 9 se denomina "Mercancías y operaciones no clasificadas en otro rubro de la CUCI" en lugar de "Oro no monetario", tal como lo describe la tabla. Sin embargo, solo esta categoría de producto está disponible en UNCTADStat (las otras categorías siendo "Paquetes postales no clasificados según su naturaleza", “Operaciones y mercancías especiales no clasificadas según su naturaleza" y “Monedas (excepto de oro) que no tengan curso legal"). Por esta razón, se tomó la decisión de asimilar la categoría "Oro monetario" al capítulo 9. En la descripción del capítulo 5, "n.e.p." significa "no especificado en otra parte".

En consecuencia, la especialización regional de Colombia se vería afectada principalmente a nivel de estos dos capítulos. Además, los productos alimenticios y los animales vivos (capítulo 0 ) incluyen 8 categorías que pierden sus ventajas comparativas, contra solamente una categoría que adquiere ventajas comparativas. Por lo tanto, la ampliación de la Alianza del Pacífico también cuestionaría la especialización regional de Colombia en materia de productos agropecuarios.

Lo anterior debería llamar la atención de los representantes colombianos en las negociaciones de conformación de la Alianza del Pacífico+4. En efecto, según la última Gran Encuesta Integrada de Hogares -GEIH- del Departamento Administrativo Nacional de Estadística -DANE-, el sector agropecuario proporcionó el $17 \%$ de los empleos totales en Colombia durante el periodo 12/17 - 02/18, y hasta el 64\% de los empleos en las zonas rurales del país. Cuestionar la especialización regional de Colombia en materia de productos agropecuarios, mediante la liberalización del comercio involucrada por la Alianza del Pacífico+4, equivale a cuestionar las fuentes de ingresos de una parte significativa de los hogares de las zonas rurales. Además, ya en estas zonas más de 5 millones de personas se encuentran en situación de pobreza multidimensional. ${ }^{7}$

En consecuencia, las negociaciones para conformar la Alianza del Pacífico+4 podrían incluir la implementación de algunas medidas proteccionistas provisionales para el sector agropecuario, tal como se hizo para el Tratado de Libre Comercio entre Colombia y Estados Unidos (Stellian y Danna-Buitrago, 2017), con el fin de obtener un periodo de transición. Así, se implementaría una política económica para limitar el impacto

7 Datos del Censo Nacional Agropecuario realizado en 2014 (sitios.dane.gov.co/cna-dashboard/\#/national). 
socioeconómico potencial de la Alianza del Pacífico+4 en las zonas rurales colombianas. Esta política podría incluir, por ejemplo, mejoras en el uso de las maquinarias por parte de los productores, así como mejoras en las infraestructuras, la asistencia técnica, el financiamiento y la gestión de riesgos, con el objetivo de aumentar la competitividad y así preparar a los productores para el incremento en la competencia internacional vía la extensión de la Alianza del Pacífico.

Para terminar, la tabla 7 da cuenta de las categorías correspondientes a los cambios 1 y 4. Estas se describen de manera más detallada en el anexo. Con base en esta tabla, las entidades públicas colombianas pueden seleccionar las categorías de productos para las cuales se debería favorecer la liberalización del comercio hacia la Alianza del Pacífico+4. Asimismo, las entidades públicas colombianas pueden seleccionar las categorías para las que se deberían negociar en particular algunas medidas proteccionistas provisionales. Lo anterior, con el fin de preparar las empresas productoras de tales bienes al incremento en la competencia internacional vía la extensión de la Alianza del Pacífico. La selección planteada con anterioridad podría realizarse tomando como referente el peso de cada categoría en el Producto Interno Bruto y/o en el empleo total del país, dando prioridad a las categorías que contribuyen más al PIB/empleo. Por ejemplo, entre las categorías que pierden sus ventajas comparativas, se encuentran las categorías CUCI 641 "Papel y cartón" y 665 "Artículos de vidrio". La primera categoría representó el $0,84 \%$ de la oferta total colombiana en 2016 , y la segunda representó el 0,21\%. Cómo el papel y cartón equivale a cuatro veces los artículos de vidrio en términos de producción, se podría dar prioridad a las medidas proteccionistas provisionales que se aplicarían a la primera categoría.

\section{Tabla 7}

Productos colombianos que obtienen/pierden ventajas comparativas con la conformación de la Alianza del Pacífico+4, categorías CUCI a tres dígitos

\begin{tabular}{|c|c|c|c|c|c|c|c|c|}
\hline \multirow{2}{*}{$\begin{array}{c}\text { Capítulo } \\
\text { CUCI }\end{array}$} & \multicolumn{3}{|c|}{$\begin{array}{c}\text { Cambio } 1-\overline{\operatorname{CSC}}_{b i}^{1}<0 \text { y } \overline{C S C}_{k i}^{2}>0 \\
\text { Obtención de ventajas comparativas }\end{array}$} & \multicolumn{5}{|c|}{$\begin{array}{l}\text { Cambio } 4-\overline{\operatorname{CSC}}_{k i}^{1}>0 \text { y } \overline{\operatorname{CSC}}_{b i}^{2}<0 \\
\text { Pérdida de las ventajas comparativas }\end{array}$} \\
\hline & \multirow{2}{*}{\multicolumn{3}{|c|}{098}} & 034 & 036 & 041 & 042 & 043 \\
\hline & & & & 045 & 046 & 074 & & \\
\hline 1 & 112 & & & & & & & \\
\hline 2 & 261 & 268 & 285 & 231 & 263 & 265 & 272 & \\
\hline
\end{tabular}

8 Cálculos propios con los datos del DANE, Balances Oferta - Utilización de productos a precios corrientes (www.dane.gov.co/index.php/estadisticas-por-tema/cuentas-nacionales). 


\begin{tabular}{|c|c|c|c|c|c|c|c|c|c|c|}
\hline $\begin{array}{l}\text { Capítulo } \\
\text { CUCI }\end{array}$ & \multicolumn{5}{|c|}{$\begin{array}{c}\text { Cambio } 1-\overline{\operatorname{CSC}}_{b}^{1}<0 \text { y } \overline{\operatorname{CSC}}_{k i}^{2}>0 \\
\text { Obtención de ventajas comparativas }\end{array}$} & \multicolumn{5}{|c|}{$\begin{array}{l}\text { Cambio } 4-\overline{\operatorname{CSC}}_{b}^{1}>0 \text { y } \overline{\operatorname{CSC}}_{b i}^{2}<0 \\
\text { Pérdida de las ventajas comparativas }\end{array}$} \\
\hline 3 & 334 & 344 & & & & 322 & & & & \\
\hline 4 & 411 & & & & & & & & & \\
\hline 5 & 516 & 592 & & & & 522 & 562 & & & \\
\hline \multirow[t]{2}{*}{6} & 671 & 681 & 683 & 689 & & 634 & 641 & 658 & 662 & 665 \\
\hline & & & & & & 677 & 687 & & & \\
\hline \multirow[t]{2}{*}{7} & 713 & 714 & 718 & 723 & 731 & 724 & 726 & 728 & 733 & 746 \\
\hline & 748 & 759 & 784 & & & 761 & 763 & 764 & 785 & \\
\hline 8 & 871 & 874 & & & & 811 & & & & \\
\hline
\end{tabular}

Fuente: elaboración propia.

Nota: para la descripción de cada categoría ver el anexo.

\section{Conclusiones}

El esquema de especialización regional de Colombia se deberá ajustar frente a los nuevos posibles escenarios de liberalización de los flujos comerciales. Este artículo se focaliza en los esfuerzos dedicados a la extensión de la Alianza del Pacífico hacia Australia, Canadá, Nueva Zelandia y Singapur. Se estudia la forma que tomaría este ajuste, con base en un análisis comparativo del indicador de Ventajas Comparativas Reveladas (VCR) calculado en términos de Contribución al Saldo Comercial (CSC). De manera previa, se efectúa un análisis de las propiedades empíricas del indicador VCR-CSC respecto a otros indicadores representativos según la literatura, para explicar por qué el indicador VCR-CSC es el más adecuado para el presente estudio, según el criterio de la estacionaridad tendencial en el tiempo de las ventajas comparativas.

Entre todas las categorías estudiadas de productos (las 255 categorías de la nomenclatura CUCI), se resaltan en primera medida las categorías que muestran ventajas comparativas en lugar de desventajas comparativas, con la extensión de la Alianza del Pacífico hacia los cuatro países arriba mencionados. Estas categorías constituyen nuevas oportunidades de especialización regional para Colombia. En este mismo orden de ideas, se resaltan en segunda medida las categorías que muestran desventajas comparativas en lugar de ventajas comparativas, con la conformación de la Alianza del Pacífico+4. Estas categorías representan amenazas para el esquema actual de especialización regional de Colombia. 
Como resultado general se encuentran más amenazas que oportunidades. Asimismo, se señala que las oportunidades y las amenazas están concentradas a nivel de los Artículos manufacturados, clasificados principalmente según el material, así como a nivel de la Maquinaria y equipo de transporte. En el caso de las amenazas, estas se concentran igualmente a nivel de los Productos alimenticios y animales vivos.

Estos resultados pueden ser útiles para los participantes de las negociaciones actuales que pretenden extender la Alianza del Pacífico a un mayor número de socios comerciales. Estos podrían obtener derogaciones (provisionales) en el caso de los productos que representan amenazas para Colombia, así como una liberalización máxima para los productos que constituyen oportunidades. De la misma manera, los resultados obtenidos ayudan a comprender mejor los posibles cambios en el ambiente de las empresas localizadas en Colombia. Finalmente, el artículo resalta la calidad del indicador VCR-CSC para realizar investigaciones similares.

Como continuación del presente trabajo se podría estudiar cómo cambiarían las exportaciones y las importaciones de cada categoría de producto, para luego medir el efecto de la Alianza del Pacífico+4 sobre la producción colombiana. En otras palabras, se propone estimar las consecuencias que tendrían los ajustes en la especialización regional de Colombia, como consecuencia de la Alianza del Pacífico+4, sobre la estructura de producción del país. Esto se podría realizar, por ejemplo, mediante modelos de equilibrio parcial (Pereira et ál., 2012) o general (Corong et ál., 2017), los cuales pueden además incorporar aspectos gravitacionales (Yotov et ál., 2017). Igualmente, se podrían utilizar varios índices derivados de los flujos comerciales: ${ }^{9}$ índice de diversificación comercial, índice de intensidad comercial, índice de complementariedad comercial, índices del tipo Hummels-Klenow, etc. De esta manera, se complementaría el presente análisis comparativo de indicadores de ventajas comparativas reveladas para diagnosticar las oportunidades y restos de Colombia en materia de especialización regional frente a la futura conformación de la Alianza del Pacífico+4. De la misma manera, se podría realizar este análisis tomando como referente los otros miembros fundadores de la Alianza del Pacífico.

$9 \quad$ Una lista de estos índices y de sus modalidades de cálculo se puede consultar en el sitio internet World Integrated Trade Solution (WITS) del Banco Mundial (wits.worldbank.org/wits). 


\section{Anexo 1 \\ Descripción de productos relacionados a los efectos de la Alianza del Pacífico+4 sobre la especialización internacional de Colombia}

La tabla A.1 presenta los productos para los cuales Colombia obtiene ventajas comparativas (cambio 1) y la tabla A.2 muestra los productos para los cuales Colombia pierde ventajas comparativas (cambio 4).

\section{Tabla A. 1}

Obtención de ventajas comparativas por Colombia con la conformación de la Alianza del Pacífico+4 (categorías CUCl a tres dígitos)

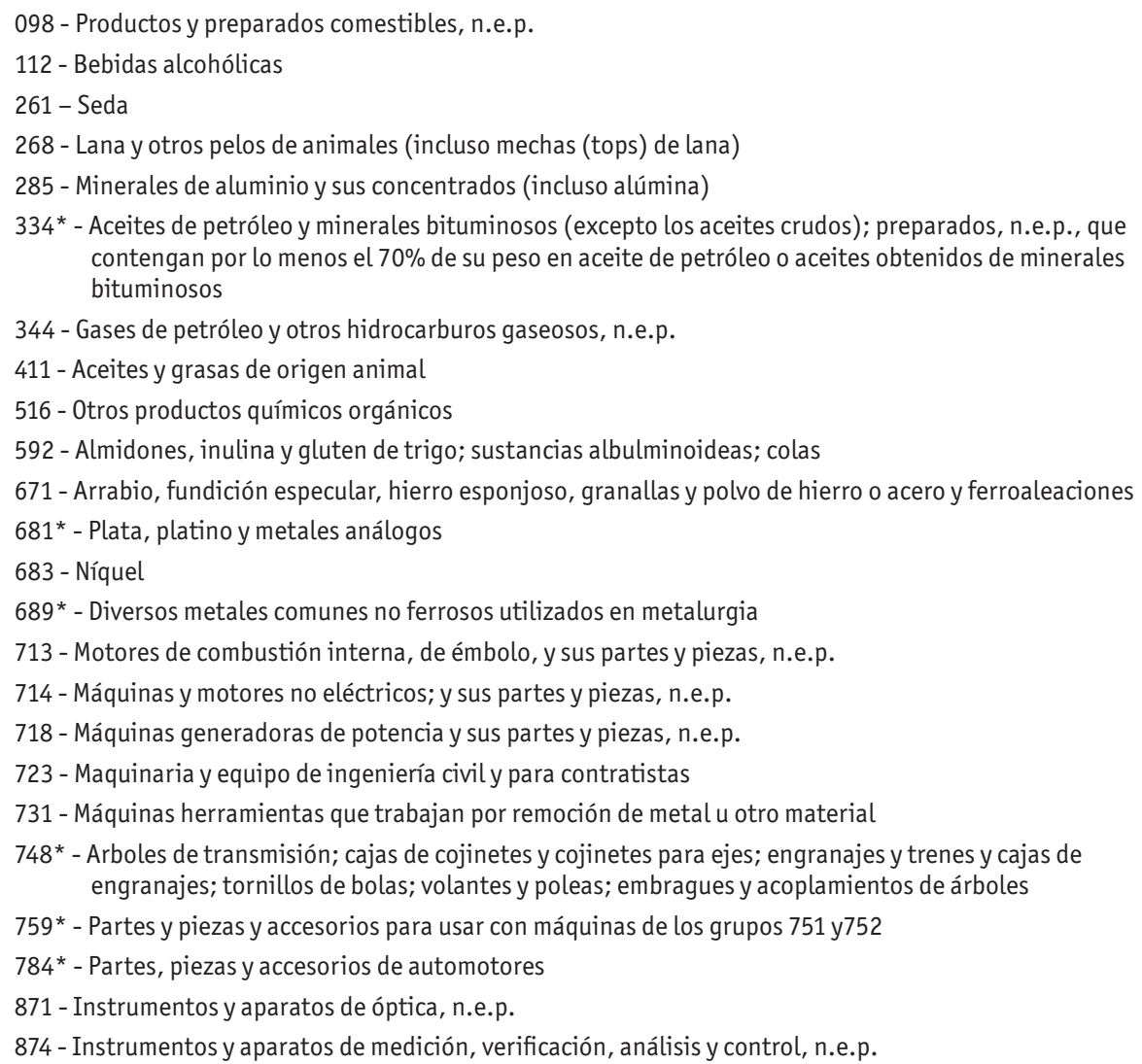

Nota: el asterisco señala que la descripción de las categorías correspondientes ha sido simplificada. "n.e.p." significa "no especificado en otra parte". 


\section{Tabla A.2}

Pérdida de ventajas comparativas por Colombia con la conformación de la Alianza del Pacífico+4 (categorías CUCl a tres dígitos)

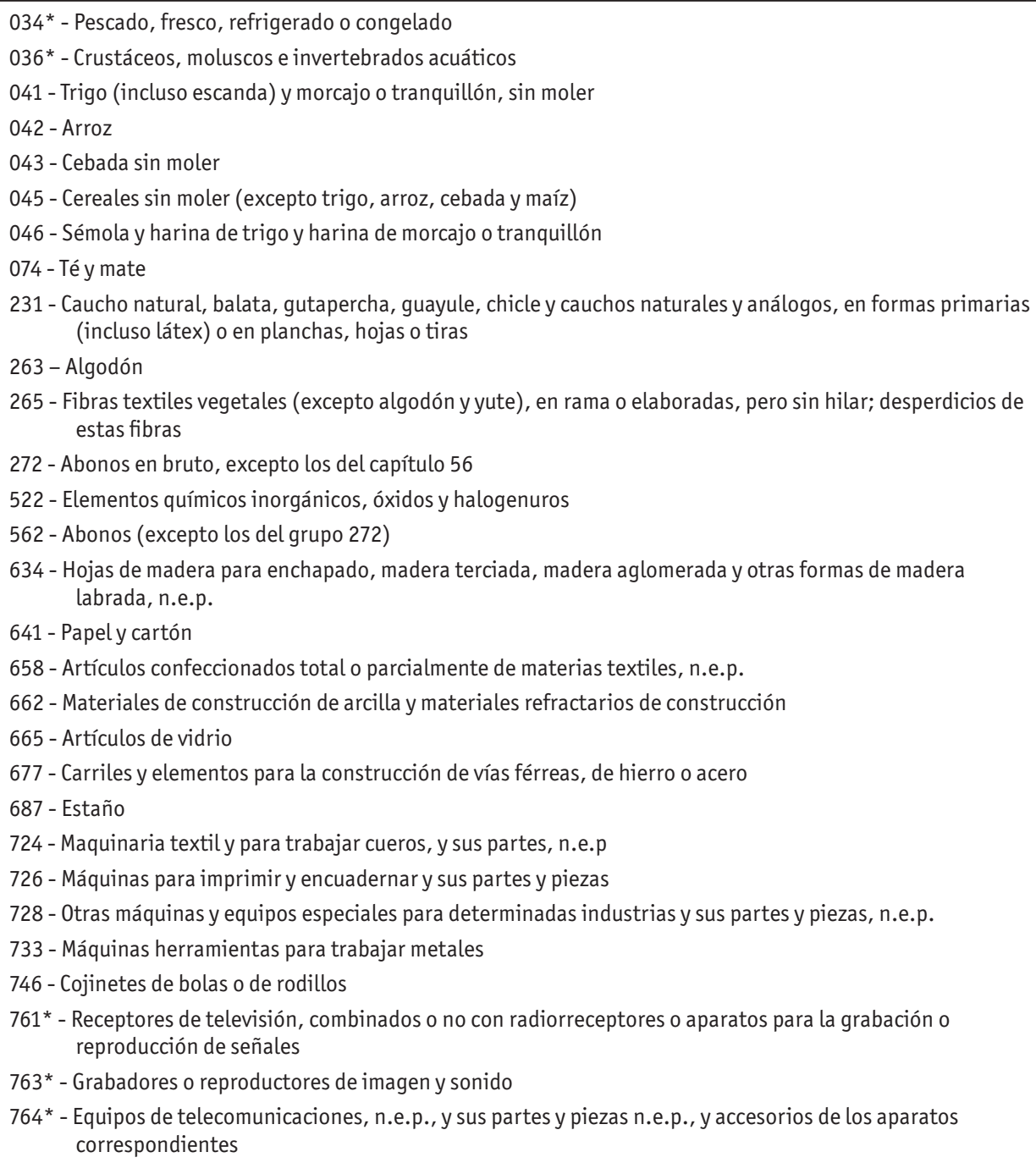

785 - Motocicletas (incluso velomotores) y velocípedos, con motor o sin él; sillones de ruedas para inválidos 811 - Edificios prefabricados

Fuente: elaboración propia.

Nota: el asterisco señala que la descripción de las categorías correspondientes ha sido simplificada. "n.e.p." significa "no especificado en otra parte". 


\section{Referencias}

Alonso, J. C. y Martínez, D. A. (2017). Impacto del precio del petróleo sobre el PIB de los países de la Alianza del Pacífico. Revista Finanzas y Política Económica, 9(2), 249-264. http:// dx.doi.org/10.14718/revfinanzpolitecon.2017.9.2.3

Arbeláez, D. y Rosso, J. (2016). Efectos estacionales en los mercados de capitales de la Alianza del Pacífico. Estudios Gerenciales, 32, 358-368. https://doi.org/10.1016/j.estger.2016.10.002

Arredondo, F., Vázquez, J. C. y De la Garza, J. (2016). Factores de innovación para la competitividad en la Alianza del Pacífico. Una aproximación desde el Foro Económico Mundial. Estudios Gerenciales, 32, 399-308. https://doi.org/10.1016/j.estger.2016.06.003

Balassa, B. (1965). Trade liberalization and 'revealed' comparative advantage. Manchester School, 33(2), 99-123. https://doi.org/10.1111/j.1467-9957.1965.tb00050.x

Castillo-Aroca, A. (2016). Depreciación del capital humano en la alianza del pacífico durante 2007-2014. Revista Ciencias Económicas, 34(1), 9-46. https://doi.org/10.15517/rce. v34i1.24498

Concha, J. R. y Gómez, 0. A. (2016). Análisis de atracción de inversión extranjera a países de la Alianza del Pacífico. Estudios Gerenciales, 32, 369-380. https://doi.org/10.1016/j. estger.2016.11.001

Corong. E., Hertel, T., McDougall, R. et ál. (2017). The Standard GTAP Model, Version 7. Journal of Global Economic Analysis, 2(1), 1-119. http://dx.doi.org/10.21642/JGEA.020101AF

Danna-Buitrago, J. P. y Stellian, R. (2014). Los retos de la integración económica latino-americana: ¿existencia o inexistencia de ventajas comparativas para la agricultura colombiana dentro de la zona ALADI? En Ágora latinoamericana: logros, obstáculos y retos de la integración regional, vol. 1. Bogotá, Asociación de Universidades de América Latina y el Caribe para la Integración (AUALCPI)/Universidad de Ciencias Aplicadas y Ambientales.

Danna-Buitrago, J. P., Stellian, R., Mercado, A. y Páez, P. (2017). Análisis retrospectivo de la integración internacional del sector manufacturero colombiano en el marco del Tratado de Libre Comercio con Estados Unidos. Bogotá: Los Libertadores.

De Saint-Vaulry, A. (2008). Base de données CHELEM-commerce international du CEPII. Document de travail CEPII, 9.

García-P. de Lema, D., Gálvez-A. E. J. y Maldonado-G., G. (2016). Efecto de la innovación en el crecimiento y el desempeño de las Mipymes de la Alianza del Pacífico: un estudio empírico. Estudios Gerenciales, 32, 326-335. https://doi.org/10.1016/j.estger.2016.07.003

González, T. (2017). Integración comercial en América Latina: estudio de políticas económicas aplicadas a través de los bloques regionales (Mercosur/Alianza del pacífico). Colección, 22(27), 137-169. 
Gutiérrez V., S., Rodríguez-R., J., Gutiérrez, L. y González-P., M. (2013). Evaluación de la justificación económica y política de la Alianza del Pacífico. Bogotá: Proexport.

Hoen, A. R. \& Oosterhaven, J. (2006). On the measurement of comparative advantage. Annals of Regional Science, 40(3), 677-691. https://doi.org/10.1007/s00168-006-0076-4

Lafay, G. (1987). Avantage comparatif et compétitivité. Économie Prospective Internationale, $29,39-52$.

Lafay, G. (1992). The measurement of revealed comparative advantages. En M. G. Dagenais \& P.-A. Muet (eds.), International Trade Modelling (pp. 209-234). London: Chapman \& Hall.

Lámbarry, F. (2016). Análisis estructural de la red económica de exportaciones e importaciones de la Alianza del Pacífico y el Mercado Común del Sur. Estudios Gerenciales, 32, 319-325. https://doi.org/10.1016/j.estger.2016.10.004

Laursen, K. (2015). Revealed comparative advantage and the alternatives as measures of international specialization. Eurasian Business Review, 5(1), 99-115. https://doi.org/10.1007/ s40821-015-0017-1

Leromain, E. \& Orefice, G. (2014). New revealed comparative advantage index: Dataset and empirical distribution. International Economics, 139, 48-70. https://doi.org/10.1016/j. inteco.2014.03.003

Maldonado B., M., y López L., S. (2017). La visión del desarrollo dentro del contexto global y regional: el regionalismo a través de la Alianza del Pacífico y la Asociación Latinoamericana de Integración 2005-2014. Desafios, 29, 13-48. http://dx.doi.org/10.12804/ revistas.urosario.edu.co/desafios/a.4902.

Martínez-C., A. (2016). Alianza del Pacífico y Mercado Común del Sur: dos enfoques de la integración económica regional. Papel Político, 21(2), 469-494. http://dx.doi.org/10.11144/ Javeriana.papo21-2.apmc.

Montoya-U., D., González-P., C. y Duarte-H., L. (2016). Situación y retos de Colombia en materia de comercio exterior, en el marco de la Alianza del Pacífico. Entramado, 12(1), 50-65. http://dx.doi.org/10.l804l/entramado.2016v12n1.23128.

Mora M., J. U. (2016). La Alianza del Pacífico y Mercosur: evidencias de convergencia económica. Estudios Gerenciales, 32, 309-318. https://doi.org/10.1016/j.estger.2016.10.005

Ovando, W., Canales, R. A. y Munguía, G. (2017). Comercio interregional de bienes manufacturados en los países de la Alianza del Pacífico desde la Teoría de Linder. Desafíos, 29(2), 169-197. http://dx.doi.org/10.12804/revistas.urosario.edu.co/desafos/a.4876

Palley, T. I. (2008). Institutionalism and new trade theory: rethinking comparative advantage and trade policy. Journal of Economic Issues, 42(1), 195-208. https://doi.org/10.108 0/00213624.2008.11507121

Pereira, C., Gómez, D. \& Herrera, L. (2012). The Colombia-Canada free trade area: A partial equilibrium simulation. Semestre Económico, 15(31), 15-42. 
Quiliconi, C. \& Salgado, R. (2017). Latin American Integration: Regionalism à la Carte in a Multipolar World? Colombia Internacional, 92, 15-41. https://doi.org/10.7440/colombiaint92.2017.01

Ramírez, J., Vásquez, M. y González, G. (2017). Comercio intrarregional de los sectores autopartes y metalmecánico del departamento de Santander con los países miembro de la Alianza del Pacífico en el periodo 2012-2015. Equidad \& Desarrollo, 28, 113-146. https:// doi.org/10.19052/ed.4086

Reyes, G. y Medina, M. (2016). Condicionantes económicas de Colombia y la Alianza del Pacífico. Tendencias, 18(1), 159-181. http://dx.doi.org/10.22267/rtend.171801.71.

Stellian, R. y Danna-Buitrago, J. P. (2017). Competitividad de los productos agropecuarios coLombianos en el marco del tratado de libre comercio con los Estados Unidos: análisis de las ventajas comparativas. Revista CEPAL, 122, 139-163.

Tremolada Á., E. (2014). ¿La Alianza del Pacífico facilita la inserción de Colombia en la región Asia - Pacífico? Papel Político, 19(2), 721-752. http://dx.doi.org/10.11144/Jave-riana. PAP019-2.apfi.

Vollrath, T. L. (1991). A theoretical evaluation of alternative trade intensity measures of revealed comparative advantage. Review of World Economics, 127(2), 265-280. https://doi. org/10.1007/BF02707986

Yeats, A. J. (1985). On the appropriate interpretation of the revealed comparative advantage index: implications of a methodology based on industry sector analysis. Review of World Economics, 121(1), 61-73. https://doi.org/10.1007/BF02705840

Yotov, Y. V., Piermartini, R., Monteiro, J.-A. \& Larch, M. (2017). An advanced guide to trade policy analysis: The structural gravity model. Ginebra: Organización Mundial del Comercio.

Yu, R., Cai, J. \& Leung P. (2009). The normalized revealed comparative advantage index. Annals of Regional Science, 43(1), 267-282. https://doi.org/10.1007/s00168-008-0213-3 